\title{
LETTER \\ MTR-Fill: A Simulated Annealing-Based X-Filling Technique to Reduce Test Power Dissipation for Scan-Based Designs
}

\author{
Dong-Sup SONG $^{\dagger \mathrm{a})}$, Jin-Ho AHN ${ }^{\dagger \dagger}$, Tae-Jin $\mathrm{KIM}^{\dagger}$, Nonmembers, and Sungho KANG ${ }^{\dagger \mathrm{b})}$, Member
}

\begin{abstract}
SUMMARY This paper proposes the minimum transition random $\mathrm{X}$ filling (MTR-fill) technique, which is a new $X$-filling method, to reduce the amount of power dissipation during scan-based testing. In order to model the amount of power dissipated during scan load/unload cycles, the total weighted transition metric (TWTM) is introduced, which is calculated by the sum of the weighted transitions in a scan-load of a test pattern and a scan-unload of a test response. The proposed MTR-fill is implemented by simulated annealing method. During the annealing process, the TWTM of a pair of test patterns and test responses are minimized. Simultaneously, the MTR-fill attempts to increase the randomness of test patterns in order to reduce the number of test patterns needed to achieve adequate fault coverage. The effectiveness of the proposed technique is shown through experiments for ISCAS' 89 benchmark circuits.

key words: low power test, scan-based test, X-filling, test application time
\end{abstract}

\section{Introduction}

Power dissipation during testing becomes a significant problem as the size and complexity of system-on-chips (SoCs) continue to grow. The excessive power during testing may cause several problems including circuit damage, yield loss, and increased product costs. Previous techniques for test power reduction have focused on reducing the shift power dissipation during test applications, which is based on four major approaches: scheduling, test vector manipulation, circuit modification, and scan chain modification.

As one of test vector modification methods, X-filling techniques have already been shown to be efficient in reducing power during testing [1]-[3]. The X-filling techniques are heuristics for assigning 0's or 1's to $\mathrm{X}$ values in test cubes in order to better achieve a specific goal. Because the $\mathrm{X}$-filling techniques utilize the $\mathrm{X}$-values that are the greater part of all the bits in the test cubes, the switching activities obtained from the X-filled test patterns can be significantly reduced. Random X-filling (R-fill) and minimum transition $\mathrm{X}$-filling (MT-fill) are widely used X-filling methods in industry [4]. When power is a consideration, it is generally better to use MT-fill. MT-fill involves filling strings of X's with the same value to minimize the number of transitions. While MT-fill minimizes power, it has the following two drawbacks:

Manuscript received September 5, 2007.

Manuscript revised November 30, 2007.

${ }^{\dagger}$ The authors are with the Department of Electrical and Electronic Engineering, Yonsei University, Korea.

${ }^{\dagger \dagger}$ The author is with the Department of Electronic Engineering, Hoseo University, Korea.

a)E-mail: songdongsup@dopey.yonsei.ac.kr

b)E-mail: shkang@yonsei.ac.kr

DOI: 10.1093/ietisy/e91-d.4.1197
- Because MT-fill may not be as effective as R-fill for detecting additional faults, the number of test patterns that are needed to achieve complete fault coverage is increased.

- Despite its effectiveness in reducing power dissipation during testing, MT-fill has the disadvantage of not considering signal transitions in the scan-unload of a test response but only minimizing the weighted transition metric (WTM) in the scan load of a test pattern.

To overcome this problem, a novel simulated annealing algorithm is proposed that is based on the $\mathrm{X}$-filling technique, which is called the minimum transition random $\mathrm{X}$ filling (MTR-fill). In order to consider signal transitions in both the scan-load of a test pattern and the scan-unload of a test response simultaneously, the proposed MTR-fill uses the total weighted transition metric (TWTM) as a cost function of the simulated annealing process. The TWTM is calculated by the sum of weighted transitions in the test pattern and the corresponding test response. The new solution of the simulated annealing process is determined so that the randomness of the intermediate test pattern is increased. If the TWTM cost of the new solution is less than that of the current solution, the new solution is accepted as the best solution. Therefore, with the simulated annealing process, test patterns generated by the proposed MTR-fill obtain appropriate fault coverage with smaller-sized test patterns and lower amounts of testing power dissipated compared with those required by MT-fill.

\section{Basic Idea}

Scan shifting power dissipation includes not only the power dissipation that is produced during the scan-load of a test pattern, but also the power dissipation that is produced during the scan-unload of a test response. MT-fill is clearly effective in minimizing the test power dissipation that is produced during the scan load. However, since MT-fill does not consider signal transitions during the scan unload, minimization of the scan shifting power dissipation cannot be guaranteed when carrying out a scan test. Consider the following example.

Assume that a scan chain test cube $t_{i}$ of the circuit shown in Fig. 1 is as follows: $t_{i}=\left\{F_{1}, F_{2}, F_{3}, F_{4}, F_{5}\right\}=$ XXXX1. In addition, $t_{1}=11111$ and $t_{2}=10111$ are scan chain test patterns that are obtained after X-filling on the scan chain test cube of $t_{i}$. Here, $t_{1}$ is the MT-filled scan 


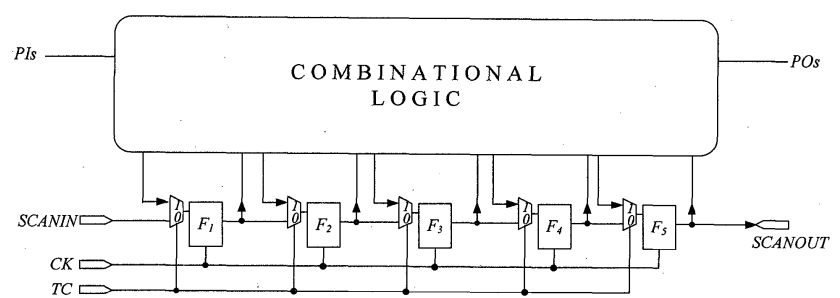

Fig. 1 Example of a full-scan circuit.

chain test pattern. The WTM metric models the fact that the scan in power for a given scan chain test pattern depends not only on the number of transitions in the pattern but also on their relative positions. Consider a scan chain of length $L$ and a scan chain test pattern $t_{i}=\left\{t_{i, 1}, t_{i, 2}, \ldots t_{i, L}\right\}$ where $t_{i, j}$ represents the $j$-th bit of $t_{i}$. The WTM for $t_{i}$ is given by:

$$
W T M\left(t_{i}\right)=\sum_{j=1}^{L-1}(L-j) \cdot\left(t_{i, j} \oplus t_{i, j+1}\right)
$$

According to the definition of WTM, the WTM of $t_{1}$ is 0 and WTM of $t_{2}$ is 3 . During the scan-load, $t_{1}$ is expected to have less power dissipation than $t_{2}$. However, this does not mean that $t_{1}$ is more power-effective than $t_{2}$. This is because scan shifting consists of both a scan-load and a scan-unload. Assume that when $t_{1}$ and $t_{2}$ are applied to the circuit the scan chain test responses, $r_{1}=01010$ and $r_{2}=00111$, are obtained, respectively. During the scan-unload, the WTM of $r_{1}$ is calculated to be 10 , and WTM of $r_{2}$ is calculated to be 2 . As a result, the MT-filled test pattern, $t_{1}$, has a total of 10 weighed signal transitions during scan shifting, whereas $t_{2}$ has only 5 weighted signal transitions. by:

The randomness of a scan chain test pattern $t_{i}$ is given

$$
\text { randomness of } t_{i}=\frac{\min \left(N_{1}\left(t_{i}\right), N_{0}\left(t_{i}\right)\right)}{L} .
$$

Here, $N_{1}\left(t_{i}\right)$ is the number of " 1 " bits in the scan chain test pattern $t_{i}$ and $N_{0}\left(t_{i}\right)$ is the number of " 0 " bits in the scan chain test pattern $t_{i}$. The randomness of a test pattern indicates the probability that it will detect additional faults with the test pattern when it is reversely fault simulated. As a test pattern increases randomness, the chance of detecting additional faults is increased. Therefore, if the randomness of each test pattern is increased, the required amount of test patterns that are needed can be reduced to achieve adequate fault coverage. According to the definition of randomness, the randomness of $t_{1}$ is 0 , and the randomness of $t_{2}$ is 0.2 . Because $t_{2}$ has higher randomness than $t_{1}$, which is $\mathrm{X}$-filled by the MT-fill algorithm, it is more likely that $t_{2}$ will detect additional faults than $t_{1}$.

\section{Minimum Transition Random X-Filling Algorithm}

Figure 2 shows the proposed MTR-fill algorithm. The MTR-fill is implemented with simulated annealing processes. As the outermost loop (line 4) is iterated, the cost

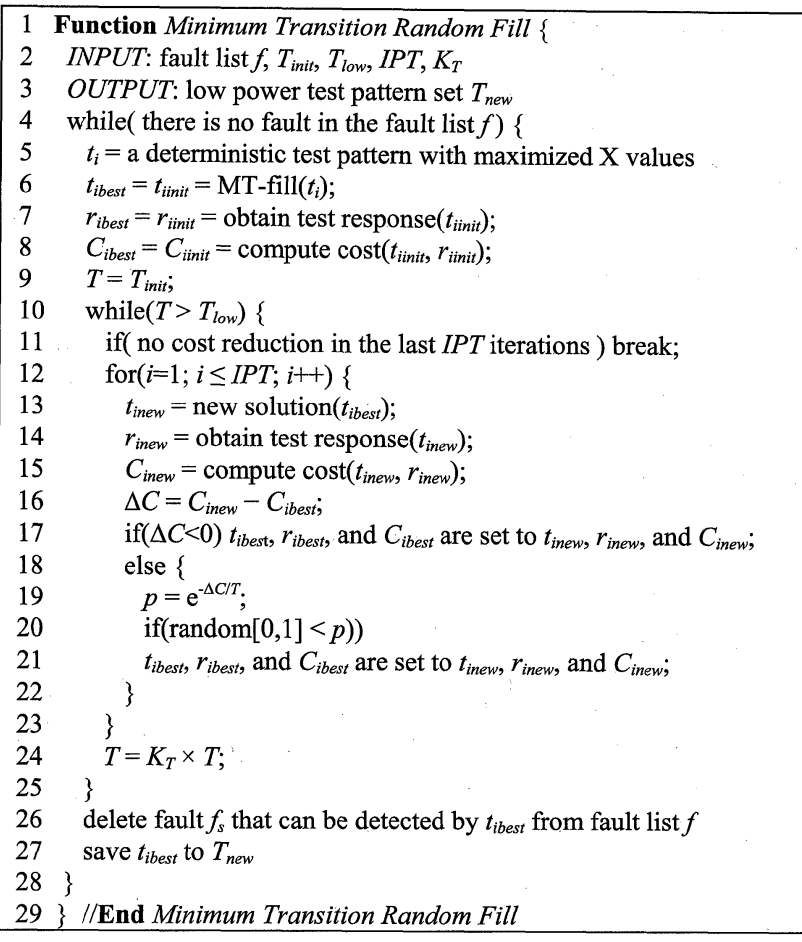

Fig. 2 The proposed MTR-fill algorithm.

of the TWTM is minimized by appropriately assigning 0 or 1 to the X-value that exists in the test cube $t_{i}$. $t_{i}$ is a deterministic test cube (line 5) that is generated by ATPG, so that the undetermined inputs remain $\mathrm{X}$-values. The initial test pattern $t_{\text {iinit }}$ and the initial test response $r_{\text {iinit }}$ are the initial solutions of the annealing process, which are determined using $t_{i}$. The initial test pattern $t_{i i n i t}$ is derived by MT-filling the X-values of $t_{i}$ (line 6), and the initial test response $r_{\text {init }}$ is the test response of the circuit on $t_{\text {init }}$ (line 7). The proposed simulated annealing algorithm uses the TWTM with a pair consisting of a test pattern and a test response as a cost function in order to minimize the scan shifting power dissipation.

The TWTM of a pair consisting of a test pattern $t_{i}$ and test response $r_{i}$ is defined as follows.

$$
\operatorname{TWTM}\left(t_{i}\right)=\sum_{j=1}^{L-1}\left(t_{i, j} \oplus t_{i, j+1}\right) \times j+\sum_{j=1}^{L-1}\left(r_{i, j} \oplus r_{i, j+1}\right) \times j
$$

Here, $L$ represents the length of the scan chain, while $t_{i, j}$ and $r_{i, j}$ signify the $j$-th bit of $t_{i}$ and $r_{i}$, respectively.

The initial cost $C_{\text {inint }}$. is calculated using $t_{\text {init }}$ and $r_{\text {init }}$ (line 8). The middle loop modifies the control parameter of the simulated annealing algorithm, which is gradually lowered as the annealing process proceeds. Within the inner loop, new solutions, $t_{\text {inew }}$ and $r_{\text {inew }}$, are generated with a constant control parameter value by the following method (line 13, line 14)

- $t_{\text {inew }}$ : Define the bit positions that are assigned to the $\mathrm{X}$-value in the deterministic test cube $t_{i}$ obtained from 
line 4 as free positions. $t_{\text {inew }}$ is the test pattern that is derived by flipping the $k$-th bit value of $t_{i b e s t}$. At this time, $k$ is randomly selected from the free positions.

- $r_{\text {inew }}$ : A test response pattern is acquired by applying $t_{\text {inew }}$ on the circuit under test.

The cost $C_{\text {inew }}$ is calculated using the cost function TWTM on the new solution $t_{\text {inew }}$ and $r_{\text {inew }}$ (line 15). If $t_{\text {inew }}$ and $r_{\text {inew }}$ produce the minimum cost of all the solutions, $t_{\text {inew }}$ and $r_{\text {inew }}$ are accepted as $t_{\text {ibest }}$ and $r_{\text {ibest }}$ (line 17). New solutions are either accepted or rejected depending on the acceptance criterion defined in the simulated annealing algorithm (line 19 - line 21). Here, random[0,1] is an uniform random number between 0 and 1 (line20). When the final best solution $t_{i b e s t}$ is determined by the annealing process, all faults that are detected by $t_{\text {ibest }}$ are eliminated from the fault list $f$ (line 26). The above process is repeated until there are no more faults in the fault list $f$.

\section{Experimental Results}

In this section, the efficiency of the proposed MTR-fill is demonstrated for the ISCAS' 89 benchmark circuits. During the simulated annealing process, the parameters are set up as $T_{\text {init }}=5.0, T_{\text {low }}=0.1, K_{t}=0.9$, and $I P T=500$. In the ATPG process, a random pattern testing session is not included and all test patterns are generated with a deterministic test pattern generation session by targeting the entire single stuck-at fault. The generated test patterns are compacted by the reverse order fault simulation. Each benchmark circuit was considered as a full scan circuit with a single scan chain.

Table 1 presents the results of the proposed MTR-fill algorithm, together with those by R-fill, 0-fill, and MT-fill. The 'Fault Cov.' column and the 'No. of Test' columns show the fault coverage obtained for stuck-at faults and the number of test patterns needed to acquire the corresponding fault coverage for each X-filling heuristic. The ' $P_{\text {ave }}$ ', and the ' $P_{\text {peak }}$ ' columns represent the average scan shifting power and the peak scan shifting power, respectively. These are given by the following equations.

$$
\begin{gathered}
P_{\text {ave }}=\frac{\sum_{i=1}^{N} T W T M\left(t_{i}\right)}{N} \\
P_{\text {peak }}=\max _{1 \leq i \leq N} T W T M\left(t_{i}\right)
\end{gathered}
$$

The last column shows the effects of test length on the proposed MTR-fill technique. The 'Test redu.' column is calculated by the ratio of the number of reduced test patterns over the number of test patterns that are required in the MTfill technique. The proposed MTR-fill attempts to increase the randomness of the test patterns by flipping the current logic value of a randomly selected bit during the simulated annealing process. As a consequence, the proposed method reduces the test length by $18.1 \%$ in comparison with those that use the MT-fill.

Table 2 compares the test power reduction of the proposed MTR-fill to that of previous works. Note that the test power reduction found in the previous work indicates only

\begin{tabular}{|c|c|c|c|c|c|c|c|c|c|c|c|c|c|c|}
\hline \multirow[b]{2}{*}{ Circuit } & \multirow[b]{2}{*}{$\begin{array}{l}\text { Fault } \\
\text { Cov. } \\
(\%)\end{array}$} & \multicolumn{3}{|c|}{ R-fill } & \multicolumn{3}{|c|}{0 -fill } & \multicolumn{3}{|c|}{ MT-fill } & \multicolumn{4}{|c|}{ Proposed MTR-fill } \\
\hline & & $\begin{array}{l}\text { No. of } \\
\text { Test }\end{array}$ & $P_{\text {ave }}$ & $P_{\text {peak }}$ & $\begin{array}{l}\text { No. of } \\
\text { Test }\end{array}$ & $P_{\text {ave }}$ & $P_{\text {peak }}$ & $\begin{array}{l}\text { No. of } \\
\text { Test }\end{array}$ & $P_{\text {ave }}$ & $P_{\text {peak }}$ & $\begin{array}{c}\text { No. of } \\
\text { Test }\end{array}$ & $P_{\text {ave }}$ & $P_{\text {peak }}$ & $\begin{array}{l}\text { Test } \\
\text { redu. } \\
(\%)\end{array}$ \\
\hline s5378 & 99.13 & 270 & 14132 & 16598 & 638 & 4225 & 6291 & 491 & 3361 & 5413 & 428 & 3026 & 5051 & 12.8 \\
\hline s9234 & 93.48 & 425 & 21895 & 25296 & 965 & 11168 & 13046 & 490 & 10470 & 13524 & 454 & 7085 & 9833 & 7.3 \\
\hline s13207 & 98.46 & 501 & 203713 & 225507 & 1711 & 90418 & 120941 & 589 & 87059 & 113070 & 546 & 52804 & 81241 & 7.3 \\
\hline $\mathrm{s} 15850$ & 96.68 & 474 & 141005 & 159914 & 1671 & 57910 & 83412 & 577 & 55441 & 81666 & 484 & 29741 & 55682 & 16.1 \\
\hline s35932 & 99.19 & 79 & 1379144 & 1595691 & 589 & 51520 & 83176 & 547 & 33240 & 79820 & 324 & 31424 & 75850 & 40.8 \\
\hline s38417 & 99.47 & 1031 & 1232412 & 1355437 & 2573 & 227416 & 281984 & 2263 & 174333 & 269912 & 1934 & 127947 & 213967 & 14.5 \\
\hline s38584 & 95.85 & 704 & 1014658 & 1079256 & 1922 & 541275 & 574792 & 1348 & 470685 & 557567 & 973 & 302689 & 381214 & 27.8 \\
\hline Ave. & 97.47 & 498 & 572423 & 636786 & 1438 & 140562 & 166235 & 901 & 119227 & 160139 & 735 & 79245 & 117548 & 18.1 \\
\hline
\end{tabular}

Table 1 Experimental results of the proposed MTR-fill technique.

\begin{tabular}{|c|c|c|c|c|c|c|c|c|c|c|c|}
\hline \multirow[b]{3}{*}{ Circuit } & \multicolumn{4}{|c|}{ Power reduction compared to MT-fill } & \multicolumn{3}{|c|}{ Power reduction compared to R-fill } & \multicolumn{4}{|c|}{ Power reduction compared to 0 -fill } \\
\hline & \multicolumn{2}{|c|}{ Test smoothing [1] } & \multicolumn{2}{|c|}{ Proposed MTR-fill } & \multirow{2}{*}{\begin{tabular}{|c|} 
SB-Filling [2] \\
$P_{\text {peak }}$ \\
redu. \\
$(\%)$ \\
\end{tabular}} & \multicolumn{2}{|c|}{ Proposed MTR-fill } & \multicolumn{2}{|c|}{ MTC-Filling [3] } & \multicolumn{2}{|c|}{ Proposed MTR-fill } \\
\hline & $\begin{array}{l}P_{\text {ave }} \\
\text { redu. } \\
(\%)\end{array}$ & $\begin{array}{c}P_{\text {peak }} \\
\text { redu. } \\
(\%)\end{array}$ & $\begin{array}{c}P_{\text {ave }} \\
\text { redu. } \\
(\%)\end{array}$ & $\begin{array}{l}P_{\text {peak }} \\
\text { redu. } \\
(\%)\end{array}$ & & $\begin{array}{c}P_{\text {ave }} \\
\text { redu. } \\
(\%)\end{array}$ & $\begin{array}{c}P_{\text {peak }} \\
\text { redu. } \\
(\%)\end{array}$ & $\begin{array}{l}P_{\text {ave }} \\
\text { redu. } \\
(\%)\end{array}$ & $\begin{array}{l}P_{\text {peak }} \\
\text { redu. } \\
(\%)\end{array}$ & $\begin{array}{l}P_{\text {ave }} \\
\text { redu. } \\
(\%)\end{array}$ & $\begin{array}{c}P_{\text {peak }} \\
\text { redu. } \\
(\%)\end{array}$ \\
\hline s5378 & 12.9 & 23.8 & 10.0 & 6.7 & 74.2 & 78.6 & 69.6 & 18.07 & 2.92 & 28.4 & 19.7 \\
\hline s9234 & 11.8 & 13.7 & 32.3 & 27.3 & 43.8 & 67.6 & 60.8 & 40.31 & 8.46 & 36.6 & 24.6 \\
\hline s13207 & 17.6 & 42.4 & 39.3 & 28.1 & 85.3 & 74.1 & 64.0 & 34.46 & 13.85 & 41.6 & 32.8 \\
\hline s15850 & 20.3 & 58.3 & 46.4 & 31.8 & NA & 78.9 & 65.2 & 30.01 & 15.99 & 48.6 & 33.2 \\
\hline s35932 & 16.7 & 21.6 & 5.5 & 5.0 & NA & 97.7 & 95.2 & NA & NA & 39.0 & 8.8 \\
\hline s38417 & 13.5 & 38.4 & 26.6 & 20.7 & 52.5 & 89.6 & 84.2 & 39.39 & 14.93 & 43.7 & 24.1 \\
\hline s38584 & 14.5 & 15.6 & 35.7 & 31.6 & $\mathrm{NA}$ & 70.2 & 64.7 & 35.39 & 9.29 & 44.1 & 33.7 \\
\hline Ave. & 15.3 & 30.6 & 28.0 & 21.6 & 63.95 & 79.5 & 72.0 & 32.94 & 10.91 & 40.3 & 25.3 \\
\hline
\end{tabular}

Table 2 Comparison results of test power reduction with previous works. 
the scan-load power reduction. However, the test power reduction of the proposed MTR-fill technique includes both the scan-load power reduction and the scan-unload power reduction. The proposed method has achieved an average reduction of $28 \%$ in $P_{\text {ave }}$ and $21.6 \%$ in $P_{\text {peak }}$ compared with the MT-fill technique. As shown in Table 2, the MTR-fill of the $\mathrm{X}$-values leads to a more significant savings in average and peak power dissipation than in previous work.

\section{Conclusions}

In this paper, a new X-filling technique, called MTR-fill, is proposed in order to address the problem of excessive power dissipation during scan testing. During the simulated annealing process, the MTR-fill reduces the number of signal transitions in a scan-load test pattern and a scanunload test response simultaneously by appropriately assigning X-values to 0's or 1's. Simultaneously, the proposed MTR-fill attempts to increase the randomness of the test pattern by flipping the current logic value of a randomly selected bit to reduce the number of test patterns needed to achieve adequate fault coverage. Experimental results show that lower test power dissipation and a small number of test patterns are required compared with those needed for the MT-fill method. The proposed MTR-fill method offers a feasible solution to the power dissipation problem by reducing the test application time in scan-based logic testing.

\section{References}

[1] T. Hayashi, N. Ikeda, T. Shinogi, H. Takase, and H. Kita, "Low power oriented test modification and compression techniques for scan based core testing," IEEE Proc. Asian Test Symposium, pp.327-332, 2006.

[2] N. Badereddine, P. Girard, S. Pravossoudovitch, C. Landrault, A. Virazel, and H.J. Wonderlich, "Minimizing peak power consumption during scan testing: Structural technique for don't care bits assignment," IEEE Proc. Research in Microelectronics and Electronics, $\mathrm{Ph}$. D., pp.65-68, 2006.

[3] J. Song, H. Yi, D. Hwang, and S. Park, "A compression improvement technique for low-power scan test data," IEEE Proc. TENCON, pp.14, 2006.

[4] K.M. Butler, J. Saxena, T. Fryars, G. Hetheringon, A. Jain, and J. Lewis, "Minimizing power consumption in scan testing: Pattern generation and DFT techniques," IEEE Proc. Int. Test Conf., pp.355-364, 2004. 